\title{
REVIEW
}

\section{Neurological complications of coeliac disease}

\author{
D S N A Pengiran Tengah, A J Wills, G K T Holmes
}

Postgrad Med J 2002;78:393-398

A variety of neurological disorders have been reported in association with coeliac disease including epilepsy, ataxia, neuropathy, and myelopathy. The nature of this association is unclear and whether a specific neurological complication occurs in coeliac disease remains unproved. Malabsorption may lead to vitamin and trace element deficiencies. Therefore, patients who develop neurological dysfunction should be carefully screened for these. However, malabsorption does not satisfactorily explain the pathophysiology and clinical course of many of the associated neurological disorders. Other mechanisms proposed include altered autoimmunity, heredity, and gluten toxicity. This review attempts to summarise the literature and suggests directions for future research.

See end of article for authors' affiliations

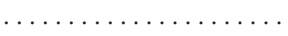

Correspondence to: Dr Holmes; GeoffreyHolmes@ compuserve.com

Submitted

6 November 2001

Accepted 26 March 2002

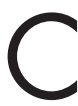
lassical coeliac disease is a gluten sensitive enteropathy in which there is small bowel villous atrophy associated with malabsorption, steatorrhoea, and weight loss. However, most patients now present with non-specific or trivial complaints and the diagnosis is only suspected from abnormalities found in routine blood tests such as anaemia or from the results of specific serological tests. Asymptomatic patients with an enteropathy characteristic of coeliac disease are labelled "silent coeliac disease" while other patients who have an apparently normal small bowel biopsy but develop typical histological features later in life are regarded as having "latent coeliac disease". These observations have led to the concept of a "coeliac iceberg" made up of a visible part of those who are diagnosed clinically and a far larger submerged portion that includes all individuals who are undiagnosed because of atypical, silent, or latent disease. ${ }^{1}$ Dermatitis herpetiformis is also a gluten sensitive disease and manifests as a blistering skin rash. Dermatitis herpetiformis and coeliac disease can be seen as part of a spectrum of illness characterised by heightened sensitivity to gluten. ${ }^{2}$ Gluten is found in foods containing wheat, barley, and rye. A gluten free diet (GFD) reverses villous atrophy and the skin changes associated with dermatitis herpetiformis. Although biopsy of small bowel and skin remain the diagnostic "gold standard" for coeliac disease and dermatitis herpetiformis respectively, antibody testing can be a useful supplementary investigation. A range of antibodies can be measured and include antireticulin, antigliadin, antiendomysial, and antitissue transglutaminase (tTG). In terms of sensitivity and specificity antiendomysial antibodies and tTG are the preferred tests for coeliac disease. Antigliadin antibodies occur in association with other conditions. ${ }^{3}$

Population screening studies have shown that coeliac disease remains under-diagnosed in adults. ${ }^{45}$ Prevalence rates are as high as 1:82 in adults in New Zealand, ${ }^{6}$ and $1.3 \%$ in healthy Swedish children. ${ }^{7}$ The variable prevalence rates among different populations may be explained by genetic factors. Ninety per cent of patients with coeliac disease carry the histocompatibility locus antigen (HLA) Dqw2. ${ }^{8}$ The prevalence of this HLA haplotype varies enormously in different ethnic groups but in the UK population is estimated at $20 \%$.

\section{DISORDERS ASSOCIATED WITH COELIAC DISEASE}

Coeliac disease is associated with conditions such as type I diabetes mellitus ${ }^{9}$ and autoimmune thyroid disease..$^{10} \mathrm{~A}$ similar association has been proposed with other autoimmune conditions such as primary biliary cirrhosis $^{11}$ and Sjogren's syndrome. ${ }^{12}$ The basis of this is unclear. A genetic mechanism has been postulated.

\section{PREVALENCE OF NEUROLOGICAL COMPLICATIONS IN COELIAC DISEASE}

The true prevalence of neurological complications in coeliac disease is difficult to estimate because of differences in study criteria and variable definitions of "neurological disorder". Moreover, most prevalence analyses have been performed in selected patient groups in tertiary referral centres. $^{13}$ Finelli et al estimated that $10 \%$ of patients with coeliac disease develop neurological complications. ${ }^{14}$

Retrospective data obtained from our hospital found 263 neurological and psychiatric conditions occurring in 189 out of 620 patients with coeliac disease (some patients had more than one disturbance) (table 1). The commonest three conditions in this series were depression (71 cases), epilepsy ( 25 cases), and migraine (20 cases). This was a heterogeneous group, including diverse conditions from self poisoning to transverse myelitis. Reunala et al found a low prevalence of neurological abnormalities in 305 patients with dermatitis herpetiformis from Finland. ${ }^{8}$ We recently took detailed histories and performed careful clinical examination in 35 patients with dermatitis herpetiformis and found no evidence for immune mediated neurological damage. ${ }^{15}$

Abbreviations: GFD, gluten free diet; $\mathrm{HLA}$,

histocompatibility locus antigen; MRI, magnetic resonance imaging; $\mathrm{TG}$, antitissue transglutaminase 
Table 1 Common neurological and psychiatric complications found in 620 patients attending the Derby coeliac clinic

\begin{tabular}{ll}
\hline Disorder & $\begin{array}{l}\text { No }(\%) \\
(\mathrm{n}=620)\end{array}$ \\
\hline Depression & $71(11.5)$ \\
Epilepsy & $25(4.0)$ \\
Migraine & $20(3.2)$ \\
Carpal tunnel syndrome & $15(2.4)$ \\
Stroke & $15(2.4)$ \\
Anxiety & $13(2.1)$ \\
Self poisoning & $11(1.8)$ \\
Myopathy & $8(1.3)$ \\
Learning difficulty & $7(1.1)$ \\
Sciatica & $6(1.0)$ \\
Meningitis & $5(0.8)$ \\
Parkinson's disease & $4(0.6)$ \\
Tension headache & $4(0.6)$ \\
Multiple sclerosis & $3(0.5)$ \\
Peripheral neuropathy & $3(0.5)$ \\
\hline
\end{tabular}

\section{PREVALENCE OF COELIAC DISEASE IN NEUROLOGICAL POPULATIONS}

Luostarinen et al detected 10 new cases of coeliac disease in neurological clinics over a three year period, accounting for $7 \%$ of the total (144) new coeliac disease cases in their hospital. ${ }^{16}$ The spectrum of neurological disorders was heterogeneous, including neuropathy (3), myopathy (1), memory impairment (2), ataxia (1), epilepsy (1), tremor (1), and parkinsonism (1). Seven of the 10 cases had pre-existing gastrointestinal symptoms or vitamin deficiencies.

In 1996, Hadjivassiliou et al looked for IgG and IgA antigliadin antibodies in two groups of neurological patients. ${ }^{17}$ The first group of 53 patients had a wide range of idiopathic neurological dysfunction and the second group of 94 patients had specific neurological diagnoses. They found a higher prevalence of positive antigliadin antibodies in the first group compared with the second $(57 \% \vee 5 \%)$. A control group of healthy blood donors had a positive rate of $12 \%$. Subsequently 26 patients from the first group consented to small bowel biopsy and nine had features consistent with coeliac disease. They have subsequently argued that IgG antigliadin antibody positivity is a sensitive marker of gluten sensitivity and may play an important part in the development of neurological illness. ${ }^{18}$ The high prevalence of antigliadin antibody positivity in their control population (12\%) remains unexplained.

Lahat and co-workers looked at the prevalence of coeliac disease in 167 children with various neurological disorders including migraine and epilepsy. ${ }^{19}$ Although they found positive IgG antigliadin antibodies in $22(13 \%)$ of 167 patients compared with three $(9 \%)$ in the control group, none had positive IgA antigliadin or antiendomysial antibodies so duodenal biopsies were not performed. They argued that there was no evidence to support a relationship between coeliac disease and neurological disorders in children and certainly no need to screen for coeliac disease in this population.

\section{NEUROLOGICAL DISTURBANCES IN COELIAC DISEASE}

The first detailed descriptive study of patients with coeliac disease (confirmed on jejunal biopsy) and a neurological deficit was published by Cooke and Smith in $1966 .{ }^{20}$ They described a group of 16 patients with a variety of neurological findings. The predominant abnormality was a sensory ataxia indicating damage to the dorsal columns in the spinal cord. In addition, three patients had evidence of cerebellar dysfunction. Since then, a number of neurological disorders have been

\section{Box 1: Disorders associated with coeliac disease}

- Type I diabetes mellitus.

- Autoimmune thyroid disease.

- Primary biliary cirrhosis.

- Sjogren's syndrome.

described in association with coeliac disease, mainly as case reports, the most frequent being cerebellar dysfunction, epilepsy, and peripheral neuropathy.

\section{Spinocerebellar degeneration and cerebellar disease}

Spinocerebellar and cerebellar disorders have been described by a number of researchers with frequent reports of ataxia in classic coeliac disease where gastrointestinal symptoms were concomitant with or preceded the neurological complications. ${ }^{21}$ There are also numerous case reports of a cerebellar syndrome where coeliac disease was a subsequent diagnosis. ${ }^{14} 223$ Myoclonic ataxia (Ramsay-Hunt syndrome) may be associated with coeliac disease $\mathrm{e}^{24-27}$ and antigliadin antibodies has been detected in the cerebrospinal fluid as well as the serum, indicating leakage through a damaged blood-brain barrier. ${ }^{26}$

The nature of the association of idiopathic cerebellar ataxia with coeliac disease is controversial. An increased incidence of coeliac disease and gluten sensitivity in patients with idiopathic cerebellar ataxia was reported by Hadjivassiliou et $a l^{28}$ and confirmed by others. ${ }^{29-31}$ However, another recent study has not supported these findings. ${ }^{32}$ Thirty two adult patients with idiopathic cerebellar ataxia were screened using antiglia$\operatorname{din}(\operatorname{IgA} / \operatorname{IgG})$, antireticulin, and antiendomysial antibodies and tTG. None of the patients were positive for any of these antibodies. It is of interest that some patients with ataxia who have a normal duodenal biopsy and raised antigliadin antibodies may also have HLA haplotypes that are associated with coeliac disease. ${ }^{28} 31$ This indicates the wide clinical manifestations of gluten sensitivity and raises the possibility that such patients might at some time develop a flat biopsy and so coeliac disease. The results for patients with hereditary ataxias have also been contradictory. One study found a high prevalence of antigliadin antibodies in patients with sporadic ataxias (seven of 26) and with hereditary ataxias (nine of 24). ${ }^{30}$ That antigliadin antibody was found in hereditary ataxias suggests that these antibodies play no part in the aetiology of the neurological dysfunction but the possibility that these contribute to the neurological degeneration still has to be entertained. However, others found no evidence of gluten sensitivity among this group. ${ }^{29}$ Further work is required to clarify differences found in the studies that might relate to patient selection or methods used to determine antibody titres.

\section{Epilepsy}

The increased prevalence of epilepsy in patients with coeliac disease is well documented. ${ }^{33} \mathrm{~A}$ high prevalence of coeliac disease has been seen in patients with a curious combination of bilateral occipital calcifications and epilepsy, ${ }^{34-37}$ mainly from Italian centres. Gobbi et al looked at two groups of such patients and found a high incidence of coeliac disease in patients with unexplained cerebral calcifications and epilepsy (24/31) and a high incidence of cerebral calcifications in patients with coeliac disease and epilepsy $(5 / 12) \cdot{ }^{38}$ Low serum folate levels were also found which might be attributable either to malabsorption or chronic anticonvulsant therapy. Interestingly, cerebral calcifications have previously been reported in other folate deficiency states. ${ }^{39}{ }^{40}$ Silica toxicity may play a part in the pathogenesis of the calcifications. ${ }^{41}$ This association of intracerebral calcifications with epilepsy and coeliac disease was not found in an Irish study. ${ }^{42}$ Although 


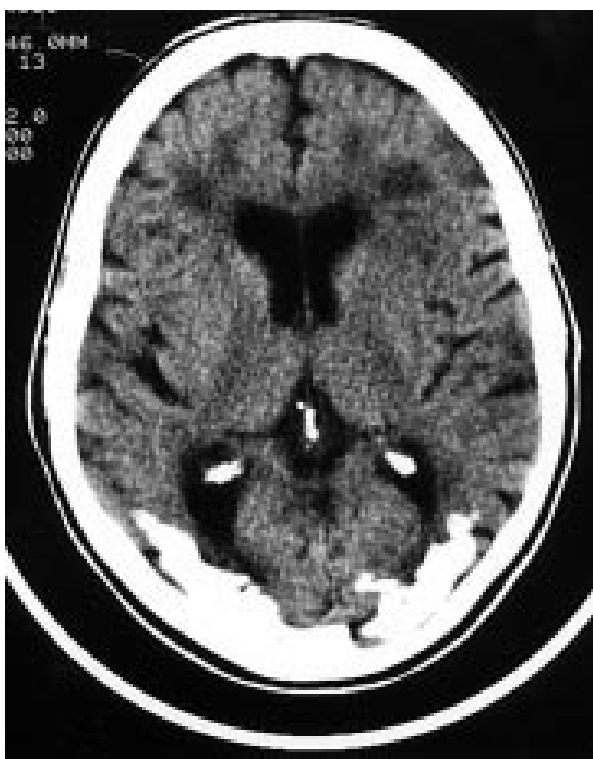

Figure 1 Computed tomogram of the brain showing bilateral occipital calcifications in a patient with epilepsy and coeliac disease.

coeliac disease occurred with increased frequency in patients with epilepsy (one in 44), no patient had cerebral calcifications on computed tomography. The geographical distribution of patients with this syndrome is unexplained for it appears to be confined largely to Italian populations (fig 1).

\section{Peripheral neuropathy}

Peripheral neuropathy in patients with established coeliac disease has been observed frequently. ${ }^{43-46}$ The neuropathy may be of axonal or demyelinating type. In two studies, the neuropathy appeared to occur because of non-compliance with a GFD and improved on reintroduction of a GFD. ${ }^{44}$ However, there was no response to a GFD in other patients.

\section{Other neuropsychiatric associations}

Many other neurological and psychiatric disorders have been described in association with coeliac disease and include myelopathy, brainstem encephalitis, ${ }^{47}$ and chronic progressive leukoencephalopathy. ${ }^{48}$ Depression is an important feature of coeliac disease, ${ }^{49}$ and appears to be more severe in those unrecognised and thus untreated by GFD or in poorly compliant patients. ${ }^{50}$ It has been suggested that schizophrenia is associated with coeliac disease ${ }^{5152}$ but this is unlikely. ${ }^{53}$ Similarly there is no firm evidence to link autism, multiple sclerosis, or dementia with gluten sensitivity and such patients should not be given a GFD. ${ }^{13}$

\section{AETIOLOGY}

\section{Role of nutritional deficiencies}

Nutritional deficiencies may play a part in the development of neurological deficits in untreated coeliac disease because of overt or occult malabsorption. Some of these are briefly discussed below. However, it is now acknowledged that these deficiencies are not sufficient factors as vitamin replacement is rarely helpful 224454 and hypovitaminosis is not always detectable. ${ }^{25}{ }^{44}$ Moreover, very often no neurological abnormality is detectable even in the presence of profound vitamin deficiency.

\section{Calcium}

Hypocalcaemia may present with neuropsychiatric features including irritability, delirium, psychosis, depression, paraesthesiae around the mouth and distal extremities, and tetany.
Osteomalacic myopathy may occur with mild hypocalcaemia, affecting proximal muscles and associated with bony pain and tenderness and a raised alkaline phosphatase. Both tetany ${ }^{55}$ and myopathy ${ }^{56}$ occur in coeliac disease.

\section{Folic acid}

Folic acid deficiency has been particularly implicated in patients with coeliac disease and epilepsy. Ventura et al described two cases of focal occipital epilepsy with cerebral calcifications and coeliac disease. ${ }^{35}$ They had previously been poorly responsive to antiepileptics. However, a GFD and folate supplementation resulted in complete electroencephalographic normalisation in one patient and marked improvement in the other. Gobbi and co-workers found low folate levels in 17 out of 19 patients with cerebral calcifications, epilepsy, and coeliac disease which they attributed to either malabsorption or anticonvulsant therapy. ${ }^{38}$ They suggested that low folate levels might be pathological as cerebral calcifications have been reported in other folate deficiency states such as in the use of antifolate treatments and radiotherapy in leukaemic children. ${ }^{39} 40$ This association was further highlighted by Bye et al who described a syndrome of bilateral occipital calcifications, epilepsy, and folic acid deficiency related to coeliac disease. ${ }^{57}$ In the two patients with myoclonic ataxia described by Lu et al, ${ }^{24}$ there was subtotal villous atrophy on jejunal biopsy, as well as reduced folate and vitamin E levels. A GFD and vitamin supplementation did not improve the neurological disorder.

\section{Pyridoxine (vitamin B6)}

Pyridoxine deficiency has been demonstrated in patients with coeliac disease. The absorption mechanism is complex. With damage to the upper duodenal mucosa, it appears that pyridoxine can be absorbed in the more distal intestine but this compensatory mechanism appears to be limited.$^{58}$ A study by Morris et al looked at the possible relationship between pyridoxine levels and neurological disorder in a group of 30 patients with adult coeliac disease ${ }^{59}$ Although pyridoxine levels were lower in patients who were non-compliant with a GFD, there was no apparent relationship between pyridoxine levels and neurological deficit. Hallert and co-workers looked at depression in patients with coeliac disease and found an improvement in symptoms after three years of GFD coupled with pyridoxine therapy. ${ }^{60}$

\section{Vitamin B12}

In a recent study of 35 newly diagnosed coeliac disease patients, vitamin B12 deficiency was found in 15 (45\%) but none had pernicious anaemia. Three complained of cold peripheries and paraesthesiae at presentation that resolved after B12 replacement. ${ }^{61}$ It has to be conceded that there is little evidence to suggest that B12 deficiency is concerned with the neurological complications of coeliac disease.

\section{Vitamin $\mathrm{E}$}

It is known that vitamin $\mathrm{E}$ is important for maintaining normal neurological function. Furthermore, in other conditions such as abetalipoproteinaemia, where hypovitaminosis $\mathrm{E}$ is secondary, vitamin E replacement may improve neurological dysfunction. ${ }^{62}$ Vitamin E deficiency has been demonstrated in patients with coeliac disease and is reversible on a GFD. ${ }^{63}$ There are reports of coeliac disease, vitamin E deficiency, and cerebellar ataxia with improvement after vitamin E replacement therapy. ${ }^{23} 6465$

\section{Biopterin}

Biopterin compounds, namely biopterin, 7,8-dihydrobiopterin, and 5,6,7,8-tetrahydrobiopterin have an important role in the metabolism of various neurotransmitters. Low levels have been demonstrated in coeliac disease and it has been 
suggested that deficiency may play a part in the development of neurological syndromes. ${ }^{66}$

\section{Carnitine}

Carnitine is required for cellular energy production and is absorbed via a transport mechanism in the small bowel. Deficiency may produce lethargy, muscular hypotonia, and encephalopathy and could contribute to the neurological manifestations of coeliac disease, although a specific investigation in this regard has not been carried out. A small study by Lerner et al compared carnitine levels in patients with coeliac disease, gastrointestinal disease with a normal small bowel biopsy, and normal subjects. ${ }^{67}$ Carnitine levels were significantly lower in patients with coeliac disease.

\section{Altered autoimmunity and inflammatory processes}

Ghezzi and co-workers described a man with coeliac disease whose small bowel biopsy revealed ulceration and an inflammatory infiltrate. ${ }^{21}$ However, two years after diagnosis, despite a GFD, his gastrointestinal symptoms deteriorated and he was given deflazacort. Gastrointestinal symptoms improved but he subsequently developed a relapsing-remitting brainstem and cerebellar syndrome considered to be due to an inflammatory process. Each relapse was treated with steroids and this appeared to improve his symptoms. Serial magnetic resonance imaging (MRI) during the course of his illness showed multiple enhancing lesions. At the start of his illness, corticosteroid administration improved the appearance of the lesions on MRI. Cerebrospinal fluid examination was initially normal but with subsequent relapses, the presence of oligoclonal IgG bands and an increased cerebrospinal fluid/serum albumin ratio was demonstrated, indicating damage to the blood-brain barrier. However, it could be argued that this patient had coeliac disease and multiple sclerosis, occurring in the same individual by chance.

An immune mediated mechanism was also suggested in a patient with chronic progressive leukoencephalopathy because of a cerebrospinal fluid lymphocytosis and raised serum immunoglobulin levels. ${ }^{48}$ A patient with treatment resistant seizures and coeliac disease was described by Rush et al.$^{68} \mathrm{He}$ was found to have an isolated central nervous system vasculitis on brain biopsy. There was clinical and radiographic improvement after treatment with prednisolone and cyclophosphamide.

\section{Gluten and antibodies to gluten}

It has been suggested that gluten is neurotoxic. Dohan proposed a correlation between cereal consumption and schizophrenia but this has not been confirmed..$^{51}$ Gluten may worsen epilepsy in coeliac disease with cerebral calcifications. ${ }^{38}$ Hadjivassiliou et $a l^{17}$ and Pellechia et $a l^{69}$ have both claimed that antigliadin antibody leads to immune mediated neurological dysfunction perhaps by damaging cerebellar Purkinje cells.

\section{TREATMENT POTENTIAL \\ Gluten free diet}

The importance of treatment with a GFD in coeliac disease is that patients benefit from normal small bowel absorption. In addition, patients with dermatitis herpetiformis experience a resolution of the rash although for reasons that are not entirely clear, the skin response is much slower than that of the gut mucosa. A GFD also reduces the risk of development of lymphoma in coeliac disease. ${ }^{70}$ Disappointingly, the potential value of a GFD in treatment or prevention remains unproved in the various neurological deficits already described. The effects of a GFD on these patients range from reversal of the dysfunction, stabilisation of the illness, to making little or no difference. It might be that there is a therapeutic window of opportunity in which commencement of a GFD is helpful. ${ }^{38}$
Hadjivassiliou and co-workers made the intriguing observation that initiation of a GFD did lead to symptom resolution in some of their patients with gluten ataxia. ${ }^{28}$ No further details were given. In established epilepsy, ${ }^{3571}$ there is limited evidence that a GFD prevents or stabilises seizures. Gobbi and co-workers suggested that a GFD seems to control seizures if started near the onset of epilepsy and early in childhood. ${ }^{38}$ Although isolated peripheral neuropathy is uncommon in coeliac disease, there is some evidence that treatment with a GFD may reverse the neuropathy. ${ }^{44}{ }^{46}$ De Santis et al described a patient with previously diagnosed schizophrenia who after investigation for weight loss and diarrhoea was found to have coeliac disease (positive antiendomysial antibodies and jejunal biopsy evidence). ${ }^{72}$ Single photon emission computed tomography showed hypoperfusion of the left frontal area. Initiation of a GFD resulted in the disappearance of psychiatric symptoms as well as improvement in the scan appearances.

Numerous studies have shown no significant benefit from a GFD. ${ }^{21}{ }^{24}$ Moreover, many patients have appeared to develop neurological complications despite adhering to a GFD. ${ }^{20}{ }^{48}$ Of the 10 cases of coeliac disease with neurological disorder detected by Luostarinen et al, ${ }^{16}$ all but one were started on a GFD. In six of the 10 patients no change occurred in the neurological symptoms. One patient with a progressive neuropathy later died of lymphoma. The neuropathy was thought to be a paraneoplastic manifestation of the lymphoma. Two patients experienced some relief of neurological symptoms, myopathy and neuropathy respectively, while a patient with epilepsy was apparently cured.

\section{Vitamin replacement}

Vitamin deficiency is discussed above. Vitamin replacement may play a part in a select group of patients but overall is of little value.

\section{Immunosuppressive treatment}

There have been a few reports of immunosuppressive treatment in patients with coeliac disease and neurological syndromes. This proved helpful in a case of vasculitis ${ }^{68}$ and in a patient with relapsing-remitting brainstem cerebellar syndrome. ${ }^{21}$ Intravenous steroids and immunoglobulins were not helpful in a case of progressive leukoencephalopathy. ${ }^{48}$

\section{CONCLUSION}

Improved case ascertainment has caused an apparent increase in the prevalence of coeliac disease. This has resulted in a changing pattern of presentation of coeliac disease with so-called atypical symptoms becoming more prominent. However, as coeliac disease becomes more commonly diagnosed, it is likely that some associated diseases may merely be a result of chance.

The increased prevalence of a variety of neurological conditions in coeliac disease suggests that patients with neurological disease are a target population who might benefit from screening and treatment. However, no firm conclusions can be made regarding the nature of the association between coeliac disease and neurological disorders as the available data are limited by the heterogeneity of patients, inconsistency of pathological findings, and lack of adequate control data. ${ }^{73} \mathrm{~A}$ GFD and vitamin supplementation may be helpful in some cases. Data regarding the value of immunosuppressive treatment are anecdotal.

It can be concluded that the majority of neurological syndromes have a chance association with coeliac disease. There may be a minority with a definite association, such as patients with certain forms of epilepsy, but further study is required to confirm this and the nature of the association. At present, it has to be conceded that a neurological disorder specifically associated with coeliac disease has not been identified. Deficiency of trace elements or vitamins might play an 
important part and this has considerable therapeutic implications. Thus far, this aspect of treatment has not been studied systematically. The role of altered autoimmunity, particularly in susceptible HLA subgroups, also merits further investigation.

Finally, with increasing recognition that coeliac disease is under-diagnosed, it would be prudent to be vigilant in all clinical settings where it might be a possible diagnosis. In the context of neurological disease, those patients with unexplained neurological dysfunction such as ataxia should have serological tests to select those who should have a small bowel biopsy for coeliac disease and treatment with a GFD. In the case of newly diagnosed patients with coeliac disease, a careful search for neurological abnormalities should form part of the initial systemic review.

\section{Authors' affiliations}

G K T Holmes, Department of Gastroenterology, Derbyshire Royal Infirmary, London Road, Derby DEI 2QY, UK

D S N A Pengiran Tengah, A J Wills, Department of Neurology

\section{REFERENCES}

1 Catassi C, Ratsch IM, Fabiani E, et al. Coeliac disease in the year 2000: exploring the iceberg. Lancet 1994;343:200-3

2 Marsh MN. The natural history of gluten sensitivity: defining, refining and re-defining. QJ Med 1995;88:9-13.

3 Rostoker G, Laurent J, Andre C, et al. High levels of lgA antigliadin antibodies in patients who have lgA mesangial glomerulonephritis but not coeliac disease. Lancet 1988;i:356-7.

4 Unsworth DJ, Brown DL. Serological screening suggests that adult coeliac disease is underdiagnosed in the UK and increases the incidence by up to $12 \%$. Gut 1994:35:61-4

5 Hin H, Bird G, Fisher P, et al. Coeliac disease in primary care: case finding study. BN 1999;318:164-7

6 Cook HB, Burt M, Collett JA, et al. Adult coeliac disease: prevalence and clinical significance. J Gastroenterol Hepatol 2000;15:1032-6.

7 Carlsson AK, Axelsson IE, Borulf SK, et al. Serological screening for celiac disease in healthy 2.5 -year-old children in Sweden. Pediatrics 2001;107:42-5

8 Reunala T. Dermatitis herpetiformis: coeliac disease of the skin. Ann Med 1998;30:416-8.

9 Holmes GKT. Coeliac disease and type 1 diabetes mellitus - the case for screening. Diabet Med 2001;18:169-77.

10 Sategna-Guidetti C, Volta U, Ciacci C, et al. Prevalence of thyroid disorders in untreated adult celiac disease patients and effect of gluten withdrawal: an Italian multicenter study. Am J Gastroenterol 2001:96:751-7.

11 Sorensen HT, Thulstrup AM, Blomqvist $P$, et al. Risk of primary biliary liver cirrhosis in patients with coeliac disease: Danish and Swedish cohort data. Gut 1999;44:736-8.

12 Iltanen S, Collin P, Korpela M, et al. Celiac disease and markers of celiac disease latency in patients with primary Sjogren's syndrome. Am J Gastroenterol 1999;94:1042-6.

13 Holmes GKT. Neurological and psychiatric complications of coeliac disease. In: Gobbi G, Andermann F, Naccarato S, et al, eds. Epilepsy and other neurological disorders in coeliac disease. London: John Libbey; 1997: 251-64

14 Finelli PF, McEntee WJ, Ambler M, et al. Adult celiac disease presenting as cerebellar syndrome. Neurology 1980;30:245-9.

15 Wills AJ, Turner B, Lock RJ, et al. Dermatitis herpetiformis and neurological dysfunction. J Neurol Neurosurg Psychiatry 2002;72:259-61.

16 Luostarinen L, Pirttila T, Collin P. Coeliac disease presenting with neurological disorders. Eur Neurol 1999;42:132-5.

17 Hadjivassiliou M, Gibson A, Davies-Jones GA, et al. Does cryptic gluten sensitivity play a part in neurological illness? Lancet 1996:347:369-71.

18 Hadjivassiliou M, Grunewald RA, Davies-Jones GA. Gluten sensitivity: a many headed hydra. BM 1999;318:1710-1

19 Lahat E, Broide E, Leshem M, et al. Prevalence of celiac antibodies in children with neurologic disorders. Pediatr Neurol 2000:22:393-6.

20 Cooke WT, Smith WT. Neurological disorders associated with adult coeliac disease. Brain 1966;89:683-722.

21 Ghezzi A, Filippi $M$, Falini A, et al. Cerebral involvement in celiac disease: a serial MRI study in a patient with brainstem and cerebellar symptoms. Neurology 1997;49:1447-50.

22 Ward ME, Murphy JT, Greenberg GR. Celiac disease and spinocerebellar degeneration with normal vitamin E status. Neurology 1985;35:1199-201

23 Beversdorf D, Moses P, Reeves A, et al. A man with weight loss, ataxia, and confusion for 3 months. Lancet 1996;347:446.

24 Lu CS, Thompson PD, Quinn NP, et al. Ramsay Hunt syndrome and coeliac disease: a new association? Mov Disord 1986;1:209-19.
25 Bhatia KP, Brown P, Gregory R, et al. Progressive myoclonic ataxia associated with coeliac disease. The myoclonus is of cortical origin, but the pathology is in the cerebellum. Brain 1995;1 18:1087-93

26 Chinnery PF, Reading PJ, Milne D, et al. CSF antigliadin antibodies and the Ramsay Hunt syndrome. Neurology 1997;49:1131-3.

27 Smith GD, Saldanha G, Britton TC, et al. Neurological manifestations of coeliac disease. J Neurol Neurosurg Psychiatry 1997;63:550

28 Hadjivassiliou M, Grunewald RA, Chattopadhyay AK, et al. Clinical, radiological, neurophysiological, and neuropathological characteristics of gluten ataxia. Lancet 1998;352:1582-5.

29 Pellecchia MT, Scala R, Filla A, et al. Idiopathic cerebellar ataxia associated with celiac disease: lack of distinctive neurological features. $J$ Neurol Neurosurg Psychiatry 1999;66:32-5.

30 Bushara KO, Goebel SU, Shill H, et al. Gluten sensitivity in sporadic and hereditary cerebellar ataxia. Ann Neurol 2001;49:540-3.

31 Burk K, Bosch S, Muller CA, et al. Sporadic cerebellar ataxia associated with gluten sensitivity. Brain 2001:124:1013-9.

32 Combarros $\mathrm{O}$, Infante J, Lopez-Hoyos $M$, et al. Celiac disease and idiopathic cerebellar ataxia. Neurology 2000;54:2346.

33 Chapman RW, Laidlow JM, Colin-Jones D, et al. Increased prevalence of epilepsy in coeliac disease. BM 1978;2:250-1.

34 Sammaritano $M$, Andermann $F$, Melanson D, et al. The syndrome of intractable epilepsy, bilateral occipital calcifications, and folic acid deficiency. Neurology 1988;38(suppl 1):239.

35 Ventura A, Bouquet F, Sartorelli C, et al. Coeliac disease, folic acid deficiency and epilepsy with cerebral calcifications. Acta Paediatr Scand 1991;80:559-62.

36 Magaudda A, Dalla Bernardina B, De Marco P, et al. Bilateral occipital calcification, epilepsy and coeliac disease: clinical and neuroimaging features of a new syndrome. J Neurol Neurosurg Psychiatry 1993;56:885-9.

37 Lea ME, Harbord M, Sage MR. Bilateral occipital calcification associated with celiac disease, folate deficiency, and epilepsy. Am Neuroradiol 1995; 16:1498-500.

38 Gobbi G, Bouquet F, Greco L, et al. Coeliac disease, epilepsy, and cerebral calcifications. The Italian Working Group on Coeliac Disease and Epilepsy. Lancet 1992;340:439-43.

39 Kay HE, Knapton PJ, O'Sullivan JP, et al. Encephalopathy in acute leukaemia associated with methotrexate therapy. Arch Dis Child 1972;47:344-54

40 Flament-Durand J, Ketelbant-Balasse P, Maurus R, et al. Intracerebral calcifications appearing during the course of acute lymphocytic leukemia treated with methotrexate and X-rays. Cancer 1975;35:319-25.

41 Toti $\mathbf{P}$, Balestri $P$, Cano $M$, et al. Celiac disease with cerebral calcium and silica deposits: x-ray spectroscopic findings, an autopsy study. Neurology 1996;46:1088-92

42 Cronin CC, Jackson LM, Feighery C, et al. Coeliac disease and epilepsy. Q J Med 1998;91:303-8.

43 Binder HJ, Solitare GB, Spiro HM. Neuromuscular disease in patients with steatorrhoea. Gut 1967;8:605-11.

44 Kaplan JG, Pack D, Horoupian D, et al. Distal axonopathy associated with chronic gluten enteropathy: a treatable disorder. Neurology 1988; $38: 642-5$

45 Simonati A, Battistella PA, Guariso G, et al. Coeliac disease associated with peripheral neuropathy in a child: a case report. Neuropediatrics 1998;29:155-8

46 Polizzi A, Finocchiaro M, Parano E, et al. Recurrent peripheral neuropathy in a girl with celiac disease. J Neurol Neurosurg Psychiatry 2000;68:104-5.

47 4Brucke T, Kollegger $H$, Schmidbauer $M$, et al. Adult coeliac disease and brainstem encephalitis. J Neurol Neurosurg Psychiatry 1988;51:456-7

48 Beyenburg S, Scheid B, Deckert-Schluter M, et al. Chronic progressive leukoencephalopathy in adult celiac disease. Neurology 1998;50:820-2.

49 Goldberg D. A psychiatric study of patients with diseases of the small intestine. Gut 1970;11:459-65.

50 Hallert C, Derefeldt T. Psychic disturbances in adult coeliac disease. I. Clinical observations. Scand J Gastroenterol 1982;17:17-9.

51 Dohan FC. Cereals and schizophrenia data and hypothesis. Acta Psychiatr Scand 1966;42:125-52.

52 Rudin DO. The choroid plexus and system disease in mental illness. III. The exogenous peptide hypothesis of mental illness. Biol Psychiatry $1981 ; 16: 489-512$

53 Marson C, Micchetti R, Volterra V. Coeliac disease and schizophrenia. In: Gobbi G, Andermann F, Naccarato S, et al, eds. Epilepsy and other neurological disorders in coeliac disease. London: John Libbey, 1997: 239-43.

54 Muller AF, Donnelly MT, Smith CM, et al. Neurological complications of celiac disease: a rare but continuing problem. Am J Gastroenterol 1996;91:1430-5.

55 Rubinstein A, Liron $M$, Bodner $G$, et al. Bilateral femoral neck fractures as a result of coeliac disease. Postgrad Med J 1982;58:61-2.

56 Hardoff D, Sharf B, Berger A. Myopathy as a presentation of coeliac disease. Dev Med Child Neurol 1980:22:781-3.

57 Bye AM, Andermann F, Robitaille Y, et al. Cortical vascular abnormalities in the syndrome of celiac disease, epilepsy, bilateral occipital calcifications, and folate deficiency. Ann Neuro 1993;34:399-403

58 Reinken L, Zieglaver H. Vitamin B-6 absorption in children with acute celiac disease and in control subjects. J Nutr 1978;108:1562-5.

59 Morris JS, Ajdukiewicz AB, Read AE. Neurological disorders and adult coeliac disease. Gut 1970;1 1:549-54. 
60 Hallert C, Astrom J, Walan A. Reversal of psychopathology in adult coeliac disease with the aid of pyridoxine (vitamin B6). Scand J Gastroenterol 1983;18:299-304

61 Dahele A, Ghosh S. Vitamin B12 deficiency in untreated celiac disease. Am J Gastroenterol 2001:96:745-50.

62 Muller DP, Lloyd JK, Wolff OH. Vitamin E and neurological function. Lancet 1983;1:225-8.

63 Muller DP, Harries JT, Lloyd JK. The relative importance of the factors involved in the absorption of vitamin $\mathrm{E}$ in children. Gut 1974:15:966-71.

64 Mauro A, Orsi L, Mortara P, et al. Cerebellar syndrome in adult celiac disease with vitamin E deficiency. Acta Neurol Scand 1991;84:167-70.

65 Battisti C, Dotti MT, Formichi P, et al. Disappearance of skin lipofuscin storage and marked clinical improvement in adult onset coeliac disease and severe vitamin E deficiency after chronic vitamin E megatherapy. J Submicrosc Cytol Pathol 1996;28:339-44.

66 Cooke WT. The neurological manifestations of malabsorption. Postgrad Med J 1978;54:760-2.
67 Lerner A Gruener N, lancu TC. Serum carnitine concentrations in coeliac disease. Gut 1993;34:933-5.

68 Rush PJ, Inman R, Bernstein $M$, et al. Isolated vasculitis of the central nervous system in a patient with celiac disease. Am J Med 1986;81:1092-4.

69 Pellecchia MT, Scala R, Perretti A, et al. Cerebellar ataxia associated with subclinical celiac disease responding to gluten-free diet. Neurology 1999;53:1606-8.

70 Holmes GKT, Prior P, Lane MR, et al. Malignancy in coeliac disease-effect of a gluten free diet. Gut 1989;30:333-8.

71 Bardella MT, Molteni N, Prampolini L, et al. Need for follow up in coeliac disease. Arch Dis Child 1994;70:211-3.

72 De Santis A, Addolorato G, Romito A, et al. Schizophrenic symptoms and SPECT abnormalities in a coeliac patient: regression after a gluten-free diet. J Intern Med 1997;242:421-3.

73 Wills AJ. The neurology and neuropathology of coeliac disease. Neuropathol App/ Neurobiol 2000;26:493-6

\section{CALL FOR FILLERS}

The Postgraduate Medical Journal invites readers to contribute fillers for the journal. They can be on any topic likely to be of interest but we particularly welcome fillers on the following:

- Self help groups.

- Favourite books.

- Life defining moments or patients.

- Medical history.

Fillers, of around 250 words, can be emailed to the editor at pmj@btinternet.com for consideration. 